\title{
Low-dose acetylsalicylic acid use and the risk of upper gastrointestinal bleeding: A meta-analysis of randomized clinical trials and observational studies
}

\author{
Vera E Valkhoff MD PhD ${ }^{1,2}$, Miriam CJM Sturkenboom PharmD PhD ${ }^{2,3}$, Catherine Hill $\mathrm{PhD}^{4}$, \\ Sander Veldhuyzen van Zanten $\mathrm{MD} \mathrm{PhD}^{5}$, Ernst J Kuipers MD $\mathrm{PhD}^{1,6}$
}

VE Valkhoff, MCJM Sturkenboom, C Hill, S Veldhuyzen van Zanten, EJ Kuipers. Low-dose acetylsalicylic acid use and the risk of upper gastrointestinal bleeding: A meta-analysis of randomized clinical trials and observational studies. Can J Gastroenterol 2013;27(3):159-167.

BACKGROUND: Low-dose acetylsalicylic acid (LDA, $75 \mathrm{mg} /$ day to $325 \mathrm{mg} /$ day) is recommended for primary and secondary prevention of cardiovascular events, but has been linked to an increased risk of upper gastrointestinal bleeding (UGIB).

OBJECTIVE: To analyze the magnitude of effect of LDA use on UGIB risk.

METHODS: The PubMed and Embase databases were searched for randomized controlled trials (RCTs) reporting UGIB rates in individuals receiving LDA, and observational studies of LDA use in patients with UGIB. Studies were pooled for analysis of UGIB rates.

RESULTS: Eighteen studies were included. Seven RCTs reported UGIB rates in individuals randomly assigned to receive LDA $(n=22,901)$ or placebo $(n=22,923)$. Ten case-control studies analyzed LDA use in patients with UGIB $(n=10,816)$ and controls without $\operatorname{UGIB}(n=30,519)$; one cohort study reported 207 UGIB cases treated with LDA only. All studies found LDA use to be associated with an increased risk of UGIB. The mean number of extra UGIB cases associated with LDA use in the RCTs was 1.2 per 1000 patients per year ( $95 \%$ CI 0.7 to 1.8 ). The number needed to harm was 816 (95\% CI 560 to 1500 ) for RCTs and 819 (95\% CI 617 to 1119) for observational studies. Meta-analysis of RCT data showed that LDA use was associated with a 50\% increase in UGIB risk (OR 1.5 [95\% CI 1.2 to 1.8$]$ ). UGIB risk was most pronounced in observational studies (OR 3.1 [95\% CI 2.5 to 3.7]).

CONCLUSIONS: LDA use was associated with an increased risk of UGIB.

Key Words: Acetylsalicylic acid; Gastrointestinal bleeding; Gastrointestinal hemorrhage; Peptic ulcer disease; Meta-analysis

\begin{abstract}
L'utilisation d'acide acétylsalicylique à faible dose et le risque de saignement du tube digestif supérieur : une méta-analyse d'essais aléatoires et contrôlés et d'études d'observation
\end{abstract}

HISTORIQUE : L'acide acétylsalicylique à faible dose (AFD, $75 \mathrm{mg} /$ jour à $325 \mathrm{mg} /$ jour) est recommandé pour la prévention primaire et secondaire des événements cardiovasculaires, mais s'associe à une augmentation du risque de saignement du tube digestif supérieur (STDS).

OBJECTIF : Analyser la magnitude de l'effet de l'utilisation d'AFD sur le risque de STDS.

MÉTHODOLOGIE : Les chercheurs ont fouillé les bases de données de PubMed et d'Embase pour en extraire les essais aléatoires et contrôlés (EAC) rendant compte des taux de STDS chez les personnes qui prennent de l'AFD, ainsi que les études d'observation sur l'utilisation d'AFD chez les patients ayant un STDS. Ils ont regroupé les études pour analyser les taux de STDS.

RÉSULTATS : Dix-huit études ont été incluses. Sept EAC indiquaient les taux de STDS chez des personnes réparties au hasard pour recevoir de l'AFD ( $\mathrm{n}=22$ 901) ou un placebo $(\mathrm{n}=22$ 923). Dix études cas-témoin portaient sur l'analyse de l'utilisation d'AFD chez des patients ayant un STDS ( $\mathrm{n}=10$ 816) et des sujets témoins sans STDS ( $\mathrm{n}=30$ 519); une étude de cohorte faisait état de 207 cas de STDS traités seulement à l'AFD. Toutes les études ont révélé que l'utilisation d'AFD s'associe à une augmentation du risque de STDS. Le nombre moyen de cas supplémentaires de STDS associés à l'utilisation d'AFD dans les EAC s'élevait à 1,2 cas sur 1000 patients par année (95\% IC 0,7 à 1,8). Le nombre nécessaire pour nuire était de 816 (95\% IC 560 à 1500 ) dans les EAC et de 819 (95\% IC 617 à 1119$)$, dans les études d'observation. La méta-analyse des données des EAC a démontré que l'utilisation d'AFD s'associait à une augmentation de $50 \%$ du risque de STDS (RRR 1,5 [95 \% IC 1,2 à 1,8]). Le risque de STDS était plus prononcé dans les études d'observation (RRR 3,1 $95 \%$ IC [2,5 à $3,7])$.

CONCLUSIONS : L'utilisation d'AFD s'associait à une augmentation du risque de STDS. cetylsalicylic acid (ASA) at doses of $75 \mathrm{mg} /$ day to $325 \mathrm{mg} /$ day
(low-dose ASA [LDA]) is recommended for secondary preven-
tion of cardiovascular events (1) and is also commonly used for pri-
mary prevention in patients with a markedly increased risk of
cardiovascular events. LDA use has been shown to decrease the inci-
dence of cardiovascular events in patients at high cardiovascular risk
(2); however, its use is associated with an increased risk of upper
gastrointestinal bleeding (UGIB) (3-5). Peptic ulcer bleeding (PUB)
accounts for most of this increased risk (6).
It is important to be able to assess the strength of the association
between LDA use and UGIB risk because of the widespread use of
LDA, both as a prescription and as an over-the-counter medication.
The overall prevalence of LDA use among adults in the United Kingdom in 2006 was $9.6 \%$, with a much higher prevalence $(35 \%)$ in individuals older than 75 years of age (7). Several reviews and metaanalyses have estimated the risk of UGIB with ASA use; however, they have used different selection criteria or have been different types of studies (8-19). Many previous reviews have not specifically examined the risk of UGIB associated with LDA use; have not separated UGIB from lower gastrointestinal bleeding or other gastrointestinal complications such as perforation $(8,11,18-20)$; have not specifically examined ASA doses $<325 \mathrm{mg} /$ day $(12,16)$; or compared the bleeding risk associated with different doses of ASA rather than comparing the risk of bleeding in ASA users with the risk in nonusers $(13,18)$. In

${ }^{1}$ Department of Gastroenterology $\mathcal{G}$ Hepatology; ${ }^{2}$ Department of Medical Informatics; ${ }^{3}$ Department of Epidemiology, Erasmus MC - University Medical

Center, Rotterdam, The Netherlands; ${ }^{4}$ Research Evaluation Unit, Oxford PharmaGenesis ${ }^{T M}$ Ltd, Oxford, United Kingdom; ${ }^{5}$ Division of

Gastroenterology, Department of Medicine, University of Alberta, Edmonton, Alberta; ${ }^{6}$ Department of Internal Medicine, Erasmus MC - University

Medical Center, Rotterdam, The Netherlands

Correspondence: Dr Vera E Valkhoff, Erasmus MC - University Medical Center, Department of Medical Informatics, Room Ee - 2159, Dr Molewaterplein

50-60, 3015 GE Rotterdam, The Netherlands. Telephone 31-10-704-4116, fax 31-10-704-4722, e-mail v.valkhoff@erasmusmc.nl

Received for publication May 11, 2012. Accepted August 18, 2012 
addition, some studies limited inclusion to a single indication for ASA such as primary cardiovascular prevention (10) or secondary prevention (15). The majority of reviews only included randomized controlled trials (RCTs) $(8,11-15,18,20)$. However, many of the RCTs included in such reviews excluded patients at high risk for UGIB and, thus, may have underestimated the risk of bleeding associated with LDA use in real-world practice. One systematic review by García Rodríguez et al (9) included observational epidemiological studies published from 1990 to 2001, but no RCTs. Another review (16) included only casecontrol studies conducted before 1989 .

The aim of the present study was to perform a meta-analysis of prospective RCTs that compared LDA therapy with placebo in different populations, regardless of indication, duration of follow-up or duration of use, as well as observational studies that evaluated the strength of the association between LDA use and UGIB risk specifically. In addition, the absolute increase in the risk of UGIB with LDA use compared with placebo was determined from RCTs.

\section{METHODS}

\section{Study selection}

The PubMed and Embase databases were searched (entries from 1989 to 2009) for RCTs reporting UGIB (including hematemesis and/or melena) or PUB involving individuals receiving LDA or placebo; and observational studies of LDA use in cases involving UGIB or PUB, or controls. The references from previous meta-analyses and reviews were also reviewed. Searches were limited to studies published in English, French, German, Spanish, Italian or Japanese. Search results were combined and duplicates were removed. Access (Microsoft Corporation, USA) was used to screen the search results in a structured manner. Initially, study titles were screened for relevance and identified for further screening using the abstract/full text. Studies were excluded if any of the following criteria applied: all studied ASA doses were $>325 \mathrm{mg} /$ day; ASA doses were not reported; all patients were undergoing gastroprotective therapy or receiving Helicobacter pylori eradication therapy; patients were taking nonsteroidal anti-inflammatory drugs (NSAIDs); or the absence of an appropriate control group(s). Studies were also excluded if UGIB or PUB rates were not reported separately from rates of lower gastrointestinal bleeding, rates of upper gastrointestinal complications were reported as a whole rather than UGIB specifically, or data were reported only for uncomplicated peptic ulcer disease. Study selection was performed independently by two authors and decisions regarding study inclusion were reached by consensus.

\section{Data extraction}

Data regarding study populations, treatments and end points were independently extracted from each identified study by two authors and any discrepancies were resolved by consensus. For each treatment arm in the RCTs, the number of trial participants, the follow-up period and the number of patients who developed the primary end point of PUB or UGIB were recorded. Two of the RCT publications $(21,22)$ reported on melena and hematemesis separately. Only the hematemesis data from these two studies were included to minimize outcome misclassification and to avoid the possibility of double-counting UGIB events. In a sensitivity analysis, the melena data were included instead.

For the observational studies, the matching criteria, covariates in the model and adjusted OR with 95\% CI were recorded. For all studies, the mean age at baseline and the percentage of male participants were assessed. For some of the studies $(6,22-24)$, this involved referring to other published articles investigating the same study populations $(25-28)$. For studies in which age was presented in categories, patient age was inferred to be the midpoint in the range. For example, for patients between 60 and 69 years of age, a mean age of 64.5 years was inferred.

\section{Data analysis}

The rate of UGIB (per 1000 individuals per year) was calculated for each included RCT. The treatment-years weighted mean number of extra UGIB cases associated with LDA use (per 1000 individuals per year) was also calculated. The number needed to harm $(\mathrm{NNH})$ - based on the inverse of the risk difference in patients using LDA compared with patients receiving placebo - was calculated for RCTs. For observational studies, the NNH was calculated (29) using the pooled OR provided by the meta-analyses of the observational studies, given a UGIB event rate of 59 per 100,000 person-years in subjects not exposed to ASA (derived from the cohort study by Sørensen et al [30]). The NNH represents the number of patients needed to be exposed to LDA to cause UGIB in one patient.

The meta-analyses were based on the $\mathrm{OR}$, which can be interpreted as an estimate of the relative risk (RR). The OR is defined as the odds of LDA exposure among patients with UGIB divided by the odds of exposure to LDA among those without UGIB. For the observational studies, the adjusted ORs or RRs, as reported in the original article, were pooled if possible; in one of the 11 studies (31), raw outcome data were used to calculate unadjusted ORs (which may be susceptible to confounding). None of the RCTs reported an RR; therefore, the crude OR and the corresponding 95\% CI was calculated for UGIB comparing ASA therapy with placebo for each individual RCT (it was hypothesized that confounding was less of an issue in RCTs than in observational studies). To include the study by Silagy et al (32), which had four confirmed UGIB cases in the ASA group but no cases in the placebo group, 0.1 events were added to both groups, resulting in 4.1 cases in the ASA group and 0.1 case in the placebo group. This avoided an infinite OR due to null events in the placebo group. In a sensitivity analysis, 0.5 events were added to both arms.

Given the potential diversity of study designs, stratified analyses according to the two groups were performed: RCTs and observational studies. Within the observational studies, a subgroup analysis of five studies was performed investigating ASA doses $\leq 100 \mathrm{mg}$ and four studies investigating ASA doses $300 \mathrm{mg}$ to $325 \mathrm{mg}$.

To estimate an overall pooled OR, a fixed-effects model was used for the RCTs (inverse variance model) using Mix version 1.7 (BiostatXL, USA) (33). To be as conservative as possible, a random-effects model was also applied. For the observational studies, a random-effects model was used because statistical heterogeneity existed across the studies. Publication bias was examined using funnel plot asymmetry and quantified using the Egger regression test (34). All statistical tests were two sided and $\mathrm{P}<0.05$ was considered to be statistically significant.

\section{Statistical heterogeneity}

Statistical heterogeneity among the studies was tested using Cochran's $\mathrm{Q}$ statistic. For this test, a two-sided $\mathrm{P} \leq 0.10$ was considered to indicate heterogeneity. To measure the degree of heterogeneity, an $\mathrm{I}^{2}$ value was calculated, with $\mathrm{I}^{2}$ values $<30 \%$ representing a low level, $30 \%$ to $75 \%$ representing a moderate level and $>75 \%$ representing a high level of heterogeneity.

\section{Search results}

\section{RESULTS}

The PubMed and Embase database searches resulted in the identification of 2011 unique studies. The titles of these studies were screened for relevance and 709 studies were identified for further screening using the abstract/full text. A total of 688 studies were subsequently excluded for the reasons listed in Figure 1, leaving a total of 21 studies (six RCTs and 15 observational studies) eligible for inclusion. The references from previous meta-analyses and reviews were also examined (8-19) and one additional eligible RCT was identified (22). Thus, a total of 22 studies (seven RCTs and 15 observational studies) were eligible for inclusion (Figure 1). Of the 15 observational studies, 14 were case-control studies and one was a cohort study.

Of the 22 studies meeting all of the inclusion criteria, three (35-37) were suspected of reporting results from the same study populations as those included in more recently published articles $(38,39)$, and one study (40) presented additional analyses from a sample that overlapped with a previous study (39). Thus, the final number of studies included 


\section{PubMed searches}

Search 1: ("Peptic Ulcer Hemorrhage"[MeSH] OR "bleeding peptic ulcer" OR "bleeding duodenal ulcer" OR "bleeding gastric ulcer" OR (("Gastrointestinal Hemorrhage"[MeSH] OR "Melena"[MeSH] OR [Hematemesis[MeSH] OR bleeding OR hemorrhage OR haemorrhage) AND ("peptic ulcer" OR "duodenal ulcer" OR "gastric ulcer" OR gastric OR stomach OR duodenal OR duodenum OR gastroduodenal OR "upper gastrointestinal" OR "upper GI" OR nonvariceal OR "non-variceal"))) AND (acetylsalicylic acid OR aspirin OR "Anti-Inflammatory Agents, Non-Steroidal"[MeSH]) NOT review

Limits: publication date: 1989-2009; language: English, French, German, Italian, Japanese, Spanish

Search 2: acetylsalicylic acid[MeSH] AND (adverse events OR side effects OR bleed OR haemorrhage)

Limits: publication date: 1989-2009; randomized controlled trial; language: English, French, German, Italian, Japanese, Spanish

\section{Embase searches}

Search 1: 'gastrointestinal hemorrhage'/exp/mj AND ('acetylsalicylic acid'/exp/mj OR 'nonsteroid antiinflammatory agent'/exp/mj) AND ([english]/lim OR [french]/lim OR [german]/lim OR [italian]/lim OR [japanese]/lim OR [spanish]/lim) AND [article]/lim AND [1989-2009]/py

Search 2: 'acetylsalicylic acid'/exp/mj/dd_ae AND [randomized controlled trial/lim AND [article]/lim AND ([english]/lim OR [french]/lim OR [german]/lim OR [italian]/lim OR [japanese]/lim OR [spanish]/lim) AND [1989-2009]/py

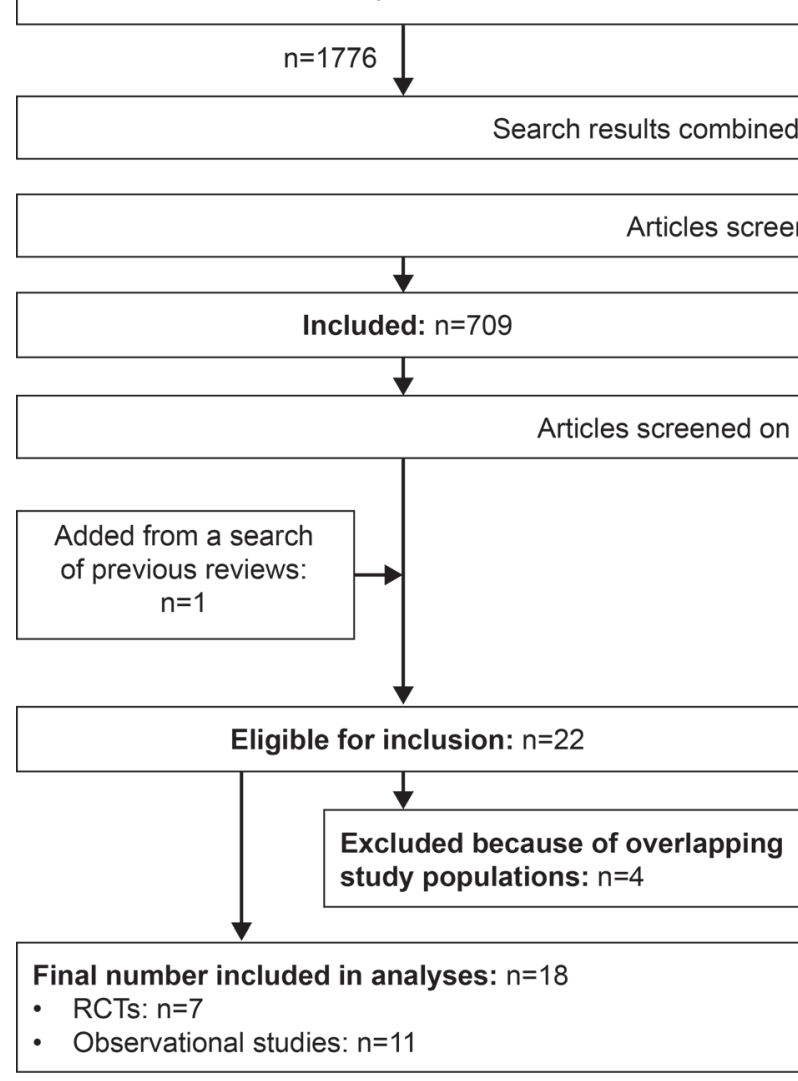
$n=619$ 
TABLE 1

Baseline characteristics of study population in seven randomized controlled trials reporting incidence of upper gastrointestinal bleeding (UGIB) in individuals randomized to receive low-dose acetylsalicylic acid (LDA) or placebo $(n=45,824)$

\begin{tabular}{|c|c|c|c|c|c|c|c|c|c|}
\hline $\begin{array}{l}\text { Author } \\
\text { (reference), } \\
\text { year }\end{array}$ & Country & Outcome of interest & Patients & Sample & $\begin{array}{c}\text { Male } \\
\text { sex, \% }\end{array}$ & $\begin{array}{c}\text { Age, } \\
\text { years, } \\
\text { mean }\end{array}$ & $\begin{array}{c}\text { Follow-up, } \\
\text { months, } \\
\text { mean }\end{array}$ & $\begin{array}{l}\text { Daily ASA } \\
\text { dose, } \\
\text { mg, mean }\end{array}$ & $\begin{array}{c}\text { UGIB } \\
\text { incidence per } \\
1000 \text { patients } \\
\text { per year } \\
\end{array}$ \\
\hline $\begin{array}{l}\text { Physicians' } \\
\text { Health Study } \\
(21), 1989\end{array}$ & USA & $\begin{array}{l}\text { Myocardial infarction, stroke, } \\
\text { cardiovascular mortality }\end{array}$ & $\begin{array}{l}\text { Male physicians } 40-84 \\
\text { years of age randomly } \\
\text { assigned to receive ASA } \\
\text { or placebo }\end{array}$ & $\begin{array}{c}\text { ASA }(n=11,037) \\
\text { placebo } \\
(n=11,034)\end{array}$ & 100 & 53.8 & 60.2 & 162.5 & $\begin{array}{c}\text { ASA: } 0.7 ; \\
\text { placebo: } 0.5\end{array}$ \\
\hline $\begin{array}{l}\text { Silagy et al } \\
(32), 1993\end{array}$ & Australia & $\begin{array}{l}\text { Adverse effects associated } \\
\text { with LDA use (part of larger } \\
\text { study on cardiovascular and } \\
\text { cerebrovascular morbidity } \\
\text { and mortality }\end{array}$ & $\begin{array}{l}\text { Individuals } \geq 70 \text { years of } \\
\text { age randomly assigned to } \\
\text { receive LDA or placebo }\end{array}$ & $\begin{array}{c}\text { ASA }(n=200) \\
\text { placebo }(n=200)\end{array}$ & 49.0 & 73.0 & 12.0 & 100 & $\begin{array}{l}\text { ASA: } 20.0 ; \\
\text { placebo: } 0.0\end{array}$ \\
\hline $\begin{array}{l}\text { Slattery et al } \\
(6), 1995 \\
\text { Farrell et al } \\
(28), 1991\end{array}$ & UK & $\begin{array}{l}\text { Adverse GI events (main } \\
\text { UK-TIA study outcomes } \\
\text { were stroke, myocardial } \\
\text { infarction and death) }\end{array}$ & $\begin{array}{l}\text { Patients with a recent TIA } \\
\text { or minor ischemic stroke } \\
\text { randomized to receive } \\
\text { ASA or placebo }\end{array}$ & $\begin{array}{c}\text { ASA }(n=806) ; \\
\text { placebo }(n=814)\end{array}$ & 72.7 & 60.0 & 48.0 & 300 & $\begin{array}{c}\text { ASA: } 5.0 ; \\
\text { placebo: } 1.5\end{array}$ \\
\hline $\begin{array}{l}\text { Diener et al } \\
(22,25), 1997\end{array}$ & $\begin{array}{l}13 \text { European } \\
\text { countries }\end{array}$ & $\begin{array}{l}\text { Stroke (fatal/non-fatal), death } \\
\text { (all cause), stroke and/or } \\
\text { all-cause death (allowed } \\
\text { first event to be counted for } \\
\text { survival analysis but } \\
\text { avoided duplicate counting). } \\
\text { Secondary outcomes: TIA } \\
\text { and other vascular events }\end{array}$ & $\begin{array}{l}\text { Patients with a recent TIA } \\
\text { or completed ischemic } \\
\text { stroke randomly assigned } \\
\text { to receive ASA or placebo }\end{array}$ & $\begin{array}{c}\text { ASA }(n=1649) \\
\text { placebo }(n=1649)\end{array}$ & 57.8 & 66.7 & 24.0 & 50 & $\begin{array}{c}\text { ASA: } 3.0 ; \\
\text { placebo: } 2.7\end{array}$ \\
\hline $\begin{array}{l}\text { Thrombosis } \\
\text { prevention } \\
\text { trial (41), } \\
1998\end{array}$ & UK & $\begin{array}{l}\text { Coronary death and fatal/ } \\
\text { nonfatal myocardial } \\
\text { infarction }\end{array}$ & $\begin{array}{l}\text { Men } 45-69 \text { years of age at } \\
\text { high risk of ischemic } \\
\text { heart disease randomly } \\
\text { assigned to receive LDA } \\
\text { or placebo }\end{array}$ & $\begin{array}{c}\text { ASA }(n=1268) ; \\
\text { placebo }(n=1272)\end{array}$ & 100 & 57.5 & $\begin{array}{c}81.6 \\
\text { (median) }\end{array}$ & 75 & $\begin{array}{c}\text { ASA: } 0.6 ; \\
\text { placebo: } 0.1\end{array}$ \\
\hline $\begin{array}{l}\text { Pulmonary } \\
\text { Embolism } \\
\text { Prevention } \\
\text { trial (42), } \\
2000\end{array}$ & $\begin{array}{l}\text { Australia, } \\
\text { New } \\
\text { Zealand, } \\
\text { South Africa, } \\
\text { Sweden, UK }\end{array}$ & $\begin{array}{l}\text { Pulmonary embolism and } \\
\text { deep vein thrombosis }\end{array}$ & $\begin{array}{l}\text { Patients undergoing } \\
\text { surgery for hip fracture } \\
\text { randomly assigned to } \\
\text { receive ASA or placebo }\end{array}$ & $\begin{array}{c}\text { ASA }(n=6679) \\
\text { placebo }(n=6677)\end{array}$ & 21.0 & 79.0 & 1.2 & $\begin{array}{c}160 \\
\text { (enteric } \\
\text { coated) }\end{array}$ & $\begin{array}{l}\text { ASA: } 328.1 ; \\
\text { placebo: } \\
231.3\end{array}$ \\
\hline $\begin{array}{l}\text { Ogawa et al } \\
(43), 2008\end{array}$ & Japan & $\begin{array}{l}\text { Atherosclerotic events } \\
\text { (including ischemic heart } \\
\text { disease, stroke, peripheral } \\
\text { arterial disease, } \\
\text { cardiovascular mortality) }\end{array}$ & $\begin{array}{l}\text { Patients } 30-85 \text { years of } \\
\text { age with type } 2 \text { diabetes } \\
\text { mellitus and no history of } \\
\text { atherosclerotic disease, } \\
\text { randomly assigned to } \\
\text { receive ASA or placebo }\end{array}$ & $\begin{array}{c}\text { ASA }(n=1262) \\
\text { placebo }(n=1277)\end{array}$ & 54.6 & 65.0 & 52.4 & 90.5 & $\begin{array}{c}\text { ASA: } 0.9 ; \\
\text { placebo: } 0.5\end{array}$ \\
\hline
\end{tabular}

ASA Acetylsalicylic acid; GI Gastrointestinal; TIA Transient ischemic attack; UK United Kingdom

\section{TABLE 2}

Baseline characteristics of study population in 11 observational studies (10 case-control, one cohort) of low-dose acetylsalicylic acid (LDA) use in patients presenting with peptic ulcer bleeding (PUB) or upper gastrointestinal bleeding (UGIB) $(n=41,542)$

\begin{tabular}{|c|c|c|c|c|c|c|c|c|c|}
\hline $\begin{array}{l}\text { Author } \\
\text { (reference), } \\
\text { year }\end{array}$ & Country & Case definition & Control definition & Sample & $\begin{array}{c}\text { Male } \\
\text { sex } \\
\%\end{array}$ & $\begin{array}{c}\text { Age, } \\
\text { years, } \\
\text { mean }\end{array}$ & $\begin{array}{l}\text { Definition of } \\
\text { LDA }\end{array}$ & $\begin{array}{l}\text { Matching } \\
\text { factors }\end{array}$ & Factors adjusted for \\
\hline $\begin{array}{l}\text { Weil et al } \\
\text { (23), } 1995 \\
\text { Langman } \\
\text { et al (26), } \\
1994\end{array}$ & UK & $\begin{array}{l}\text { Patients with } \\
\text { gastric or } \\
\text { duodenal ulcer } \\
\text { bleeding }\end{array}$ & $\begin{array}{l}\text { Hospital patients selected from } \\
\text { acute medical admissions } \\
\text { (excluding patients with acute } \\
\text { myocardial infarction, acute } \\
\text { rheumatic diseases and } \\
\text { active nonbleeding ulcers); } \\
\text { community controls selected } \\
\text { from the same primary care } \\
\text { practices as cases }\end{array}$ & $\begin{array}{l}\text { Cases } \\
(n=1121) ; \\
\text { hospital } \\
\text { controls } \\
(n=1126) ; \\
\text { community } \\
\text { controls } \\
(n=989)\end{array}$ & 55.7 & 73.6 & $\begin{array}{l}75 \mathrm{mg}, 150 \mathrm{mg} \text { or } \\
300 \mathrm{mg}, 5 \text { days/ } \\
\text { week for at least } \\
\text { the previous } \\
\text { month }\end{array}$ & $\begin{array}{l}\text { Age }( \pm 5 \\
\text { years) and } \\
\text { sex }\end{array}$ & $\begin{array}{l}\text { Other NSAID use, previous } \\
\text { ulcer or dyspepsia, } \\
\text { smoking and alcohol } \\
\text { consumption }\end{array}$ \\
\hline $\begin{array}{l}\text { Kelly et al } \\
\text { (39), } 1996\end{array}$ & USA & $\begin{array}{l}\text { Patients with } \\
\text { major UGIB } \\
\text { due to gastric } \\
\text { or duodenal } \\
\text { ulcer or } \\
\text { gastritis }\end{array}$ & $\begin{array}{l}\text { Community controls from the } \\
\text { general population }\end{array}$ & $\begin{array}{l}\text { Cases } \\
\qquad(n=550) ; \\
\text { controls } \\
(n=1202)\end{array}$ & 61.9 & $60.1^{*}$ & $\begin{array}{l}\leq 325 \mathrm{mg} / \text { day, any } \\
\text { use in the } 7 \\
\text { days before the } \\
\text { index date }\end{array}$ & $\begin{array}{l}\text { Residence, } \\
\text { sex and } \\
\text { age }( \pm 5 \\
\text { years })\end{array}$ & $\begin{array}{l}\text { Age, sex, marital status, } \\
\text { date of interview, years of } \\
\text { education, cigarette } \\
\text { smoking, coffee } \\
\text { consumption, alcohol } \\
\text { consumption, use of ASA, } \\
\text { paracetamol and NSAIDs }\end{array}$ \\
\hline
\end{tabular}


TABLE 2 - CONTINUED

Baseline characteristics of study population in 11 observational studies (10 case-control, one cohort) of low-dose acetylsalicylic acid (LDA) use in patients presenting with peptic ulcer bleeding (PUB) or upper gastrointestinal bleeding (UGIB) ( $n=41,542)$

\begin{tabular}{|c|c|c|c|c|c|c|c|c|c|}
\hline $\begin{array}{l}\text { Author } \\
\text { (reference), } \\
\text { year }\end{array}$ & Country & Case definition & Control definition & Sample & $\begin{array}{c}\text { Male, } \\
\%\end{array}$ & $\begin{array}{l}\text { Age, } \\
\text { years, } \\
\text { mean }\end{array}$ & $\begin{array}{l}\text { Definition of } \\
\text { LDA }\end{array}$ & $\begin{array}{l}\text { Matching } \\
\text { factors }\end{array}$ & Factors adjusted for \\
\hline $\begin{array}{l}\text { Lanas et al } \\
\text { (38), } 2000\end{array}$ & Spain & $\begin{array}{l}\text { Patients with } \\
\text { UGIB caused } \\
\text { by ulcers, ero- } \\
\text { sions or acute } \\
\text { mucosal lesions } \\
\text { of the upper } \\
\text { gastrointestinal } \\
\text { tract }\end{array}$ & $\begin{array}{l}\text { Hospital patients admitted for } \\
\text { reasons that were unlikely to } \\
\text { be related to NSAID treatment } \\
\text { and community controls from } \\
\text { primary care practices (one of } \\
\text { each control per case) }\end{array}$ & $\begin{array}{l}\text { Cases } \\
(n=1122) \\
\text { controls } \\
(n=2231)\end{array}$ & 69.3 & 65.2 & $\begin{array}{l}\leq 300 \text { mg/day, } \\
\text { continuous use } \\
\text { per day during } \\
\text { the previous } 7 \\
\text { days before } \\
\text { admission or the } \\
\text { day of the } \\
\text { interview }\end{array}$ & $\begin{array}{l}\text { Sex and age } \\
( \pm 5 \text { years })\end{array}$ & $\begin{array}{l}\text { Age, sex, hospital, history of } \\
\text { UGIB, peptic ulcer disease, } \\
\text { cardiovascular disease, } \\
\text { cerebrovascular disease } \\
\text { and rheumatic disease }\end{array}$ \\
\hline $\begin{array}{l}\text { Sørensen } \\
\text { et al (30), } \\
2000\end{array}$ & Denmark & $\begin{array}{l}\text { Cohort study of } \\
\text { LDA users with } \\
\text { UGIB }\end{array}$ & UGIB rate in general population & $\begin{array}{l}\text { Cases } \\
(\mathrm{n}=207 \text { in } \\
27,694 \text { LDA } \\
\text { users); con- } \\
\text { trols: NA }\end{array}$ & 49.9 & 68.1 & $\begin{array}{c}100 \mathrm{mg} / \text { day or } \\
150 \mathrm{mg} / \text { day }\end{array}$ & NA & $\begin{array}{l}\text { SIR adjusted for age, sex and } \\
\text { calendar time distribution of } \\
\text { person-years in cohort }\end{array}$ \\
\hline $\begin{array}{l}\text { de Abajo and } \\
\text { García } \\
\text { Rodríguez, } \\
\text { (44), } 2001\end{array}$ & UK & $\begin{array}{l}\text { Primary care } \\
\text { patients with } \\
\text { UGIB whose } \\
\text { specific site of } \\
\text { bleeding was } \\
\text { located in the } \\
\text { stomach or duo- } \\
\text { denum, or in } \\
\text { whom the diag- } \\
\text { nosis was pep- } \\
\text { tic ulcer }\end{array}$ & $\begin{array}{l}\text { Primary care patients with no } \\
\text { UGIB }\end{array}$ & $\begin{array}{l}\text { Cases } \\
\quad(n=1833) \\
\text { controls } \\
(n=11,500)\end{array}$ & 62.4 & 63.4 & $\begin{array}{l}\text { Various (generally } \\
75-300 \text { mg/day), } \\
\text { current use } \\
\text { defined as when } \\
\text { the supply of a } \\
\text { prescription for } \\
\text { ASA lasted until } \\
\text { the index date or } \\
\text { ended within the } \\
\text { period of } 30 \text { days } \\
\text { before the index } \\
\text { date }\end{array}$ & $\begin{array}{l}\text { Age }( \pm 1 \text { year }) \\
\text { sex and } \\
\text { calendar } \\
\text { year }\end{array}$ & $\begin{array}{l}\text { Antecedents of } \\
\text { gastrointestinal disorders, } \\
\text { smoking status, alcohol } \\
\text { consumption and use of } \\
\text { NSAIDs, anticoagulants, } \\
\text { steroids, SSRIs and } \\
\text { paracetamol }\end{array}$ \\
\hline $\begin{array}{l}\text { Stack et al } \\
(45), 2002\end{array}$ & UK & $\begin{array}{l}\text { Patients with } \\
\text { PUB admitted } \\
\text { to a single hos- } \\
\text { pital }\end{array}$ & $\begin{array}{l}\text { Hospital patients admitted for } \\
\text { reasons that would not have } \\
\text { had a major effect on use of } \\
\text { ASA or NSAIDs }\end{array}$ & $\begin{array}{l}\text { Cases } \\
(n=203) \\
\text { controls } \\
(n=203)\end{array}$ & 62.6 & 66.3 & $\begin{array}{l}\leq 300 \mathrm{mg} / \mathrm{day} \text {, any } \\
\text { use within the } \\
\text { week before } \\
\text { admission }\end{array}$ & $\begin{array}{l}\text { Age } \\
( \pm 5 \text { years }) \\
\text { and sex }\end{array}$ & $\begin{array}{l}\text { Smoking, ulcer history, } \\
\text { H pylori infection, } \\
\text { NSAID dose }\end{array}$ \\
\hline $\begin{array}{l}\text { Ibáñez et al } \\
\text { (24), } 2006 \\
\text { Laporte } \\
\text { et al (27), } \\
2004\end{array}$ & Spain & $\begin{array}{l}\text { Patients with } \\
\text { primary } \\
\text { diagnosis of } \\
\text { acute UGIB } \\
\text { from a duodenal } \\
\text { or gastric ulcer, } \\
\text { acute lesions of } \\
\text { the gastric } \\
\text { mucosa, erosive } \\
\text { duodenitis or } \\
\text { mixed lesions }\end{array}$ & $\begin{array}{l}\text { Patients admitted with non- } \\
\text { alcohol-related trauma, } \\
\text { elective surgery for nonpainful } \\
\text { disorders, and acute clinical } \\
\text { conditions believed to be } \\
\text { unrelated to ASA intake }\end{array}$ & $\begin{array}{l}\text { Cases } \\
\qquad \begin{array}{l}(n=2813) \\
\text { controls } \\
(n=7193)\end{array}\end{array}$ & 71.6 & 61.8 & $\begin{array}{l}\text { Various, any use in } \\
\text { the } 7 \text { days before } \\
\text { the index date }\end{array}$ & 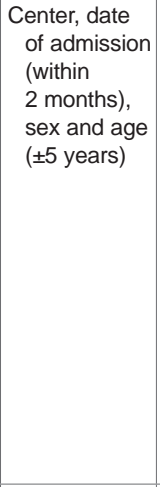 & $\begin{array}{l}\text { Clopidogrel, dipyridamole, } \\
\text { indobufen, ticlopidine, and } \\
\text { triflusal use, history of peptic } \\
\text { ulcer, diabetes mellitus, } \\
\text { heart failure, acute } \\
\text { myocardial infarction, } \\
\text { angina, stroke, transient } \\
\text { ischemic attack, intermittent } \\
\text { claudication, smoking, } \\
\text { alcohol consumption, use of } \\
\text { antacids, } \mathrm{H}_{2} \text { RAs, PPIs, } \\
\text { misoprostol, sucralfate, } \\
\text { nitrates, systemic NSAIDs, } \\
\text { topical NSAIDs, analgesics, } \\
\text { calcium-channel blockers, } \\
\text { ACE inhibitors, statins, } \\
\text { SSRIs, } \beta \text {-blockers }\end{array}$ \\
\hline $\begin{array}{l}\text { Lanas et al } \\
\text { (46), } 2006\end{array}$ & Spain & $\begin{array}{l}\text { Patients } \\
\text { hospitalized due } \\
\text { to PUB }\end{array}$ & $\begin{array}{l}\text { Hospital controls presenting for } \\
\text { reasons that would not influ- } \\
\text { ence NSAID use }\end{array}$ & $\begin{array}{l}\text { Cases } \\
\qquad \begin{array}{l}(n=2777) \\
\text { controls } \\
(n=5532)\end{array}\end{array}$ & 59.1 & 61.0 & $\begin{array}{l}\text { Various (ranging } \\
\text { from } 100 \mathrm{mg} / \mathrm{day} \\
\text { to }>1000 \mathrm{mg} / \\
\text { day), used within } \\
7 \text { days before the } \\
\text { index date }\end{array}$ & $\begin{array}{l}\text { Age }( \pm 5 \\
\text { years }) \\
\text { hospital and } \\
\text { month of } \\
\text { admission }\end{array}$ & $\begin{array}{l}\text { Age, sex, calendar semester, } \\
\text { ulcer history and use of } \\
\text { nitrates, oral anticoagulants, } \\
\text { antiplatelet agents, acid- } \\
\text { suppressing drugs, } \\
\text { NSAIDs, coxibs }\end{array}$ \\
\hline $\begin{array}{l}\text { Sakamoto } \\
\text { et al (47), } \\
2006\end{array}$ & Japan & $\begin{array}{l}\text { Patients with } \\
\text { bleeding caused } \\
\text { by a gastric or } \\
\text { duodenal ulcer or } \\
\text { gastritis, } 93.8 \% \\
\text { of whom had } \\
\text { PUB }\end{array}$ & $\begin{array}{l}\text { Community controls from } \\
\text { general population }\end{array}$ & $\begin{array}{l}\text { Cases } \\
(n=175) \\
\text { controls } \\
(n=347)\end{array}$ & 74.5 & 60.9 & $\begin{array}{l}<325 \mathrm{mg} / \text { day used } \\
\text { within } 7 \text { days } \\
\text { before the index } \\
\text { date }\end{array}$ & $\begin{array}{l}\text { Sex, age } \\
\quad( \pm 5 \text { years }) \\
\text { region }\end{array}$ & $\begin{array}{l}\text { Use of acetaminophen, } \\
\text { NSAIDs, consumption of } \\
\text { alcohol and caffeine- } \\
\text { containing beverages, } \\
\text { smoking, history of gastric or } \\
\text { duodenal ulcer, } H \text { pylori } \\
\text { status if available }\end{array}$ \\
\hline $\begin{array}{l}\text { Udd et al } \\
\qquad(48), 2007\end{array}$ & Finland & $\begin{array}{l}\text { Patients with } \\
\text { endoscopically } \\
\text { confirmed PUB }\end{array}$ & $\begin{array}{l}\text { Patients undergoing } \\
\text { investigation for dyspepsia } \\
\text { with no endoscopic evidence } \\
\text { of peptic ulcer disease }\end{array}$ & $\begin{array}{l}\text { Cases: } \\
(n=94) ; \\
\text { controls } \\
(n=94)\end{array}$ & 62.8 & 63.8 & $\begin{array}{l}\text { 50-250 mg/day, } \\
\text { taken for } \\
2 \text { weeks }\end{array}$ & $\begin{array}{l}\text { Age } \\
( \pm 5 \text { years }) \\
\text { and sex }\end{array}$ & $\begin{array}{l}\text { Ulcer history, H pylori } \\
\text { infection, NSAID use, } \\
\text { alcohol consumption, } \\
\text { smoking }\end{array}$ \\
\hline $\begin{array}{l}\text { Uppalapati } \\
\text { et al (31), } \\
2009\end{array}$ & USA & $\begin{array}{l}\text { Inpatients or } \\
\text { outpatients with } \\
\text { clinical or } \\
\text { endoscopic } \\
\text { evidence of } \\
\text { bleeding peptic } \\
\text { ulcer disease }\end{array}$ & $\begin{array}{l}\text { Inpatients or outpatients with } \\
\text { nonbleeding peptic ulcer } \\
\text { disease }\end{array}$ & $\begin{array}{l}\text { Cases } \\
(n=128) \\
\text { controls } \\
(n=102)\end{array}$ & 43.7 & 61.8 & $\begin{array}{l}\text { Various ( } 81 \mathrm{mg} / \text { day } \\
\text { to } 325 \mathrm{mg} / \text { day) }\end{array}$ & None reported & $\begin{array}{l}\text { NA: crude OR calculated in } \\
\text { the present meta-analysis }\end{array}$ \\
\hline
\end{tabular}

${ }^{*}$ Median age, years. ACE Angiotensin-converting enzyme; ASA Acetylsalicylic acid; coxibs selective inhibitors of cyclooxygenase-2; ${ }_{2} R A$ Histamine-2 receptor antagonist; H pylori Helicobacter pylori; NA Not applicable (cohort study); NSAID Nonsteroidal anti-inflammatory drug; PPI Proton pump inhibitor; SIR Standard incidence ratio; SSRI Selective serotonin reuptake inhibitor; UK United Kingdom 


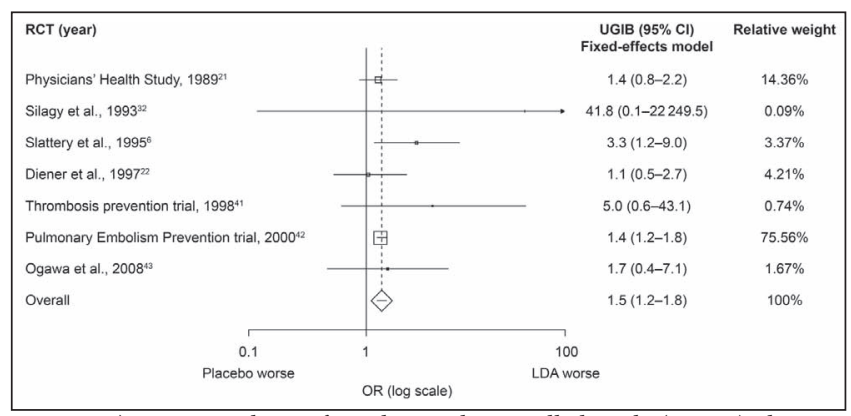

Figure 2) Meta-analysis of randomized controlled trials (RCTs) showing the association between low-dose acetylsalicylic acid (LDA) use and the risk of upper gastrointestinal bleeding (UGIB). For heterogeneity $P=0.50$; $I^{2}=0 \%$. Fixed-effects model 1.5 (95\% CI 1.2 to 1.8$) ; P<0.0001$. Random-effects model 1.5 (95\% CI 1.2 to 1.8); P<0.0001

cohort study, there were 207 UGIB cases exposed to LDA only (30). The mean age in the observational studies, weighted according to sample size, was approximately 63.3 years (range 60.1 to 73.6 years), and $64.0 \%$ of participants were male (range $43.7 \%$ to $74.5 \%$ ).

The control groups used in the 10 case-control studies varied. Three studies used a control group comprising hospital patients $(24,45,46)$, three studies included a control group obtained from primary care or the general population $(39,44,47)$, and three studies included a combination of hospital patients and community-based controls $(23,31,38)$. One study used a control group of patients with endoscopically confirmed nonulcer dyspepsia (48).

Four of the case-control studies examined the risks of PUB or UGIB associated with specific doses of ASA $(23,31,44,46)$, while the other six grouped ASA doses into one or more dose ranges $(24,38,39,45,47,48)$. The cohort study reported the standard incidence ratios (SIRs) of UGIB for doses of $100 \mathrm{mg} /$ day and $150 \mathrm{mg} /$ day, but it was unclear whether these findings were from a group of patients who were also being treated with other NSAIDs (30).

\section{Association between LDA use and UGIB}

RCTs: During follow-up, 1.3\% (288 of 22,901) of participants assigned to the ASA group developed UGIB. During the same period, $0.8 \%$ (194 of 22,923) of participants assigned to the placebo group developed UGIB.

All of the RCT publications reported increased rates of UGIB or PUB in individuals receiving LDA compared with those receiving placebo, of which two were statistically significant $(6,42)$ and five $(21,22,32,41,43)$ were not. Across the various studies, UGIB rates were in the range of 0.6 to 328.1 per 1000 patients per year in the LDA group, and 0 to 231.3 per 1000 patients per year in the placebo group. Based on this analysis, the number of extra UGIB cases associated with LDA use ranged from 0.2 to 96.8 per 1000 patients per year. The personyears weighted average number of extra UGIB cases associated with LDA use was 1.2 per 1000 patients per year ( $95 \%$ CI 0.7 to 1.8 ). The $\mathrm{NNH}$ was 816 (95\% CI 560 to 1500 ), meaning that if 816 individuals received LDA, one would have developed UGIB as a result of treatment.

After pooling the results, the OR of UGIB associated with LDA use was 1.5 (95\% CI 1.2 to $1.8 ; \mathrm{P}<0.0001$ ) (Figure 2). There was no heterogeneity $\left(\mathrm{I}^{2}=0 \%\right)$ and, because this was not significant $(\mathrm{P}=0.50)$, a fixed-effects model was applied. The result of the Egger regression test for publication bias was not significant (intercept 0.8 [95\% CI -0.3 to 1.9 ]; $\mathrm{P}=0.12$ ) and no funnel plot asymmetry was observed (Figure 3A), indicating no evidence of publication bias. The pooled estimate was mostly driven by the Pulmonary Embolism Prevention trial (42), with a relative weight of $75.6 \%$.

As mentioned previously, two of the RCTs $(21,22)$ reported on hematemesis and melena separately. When the melena data of these studies were included instead of the hematemesis data, the association

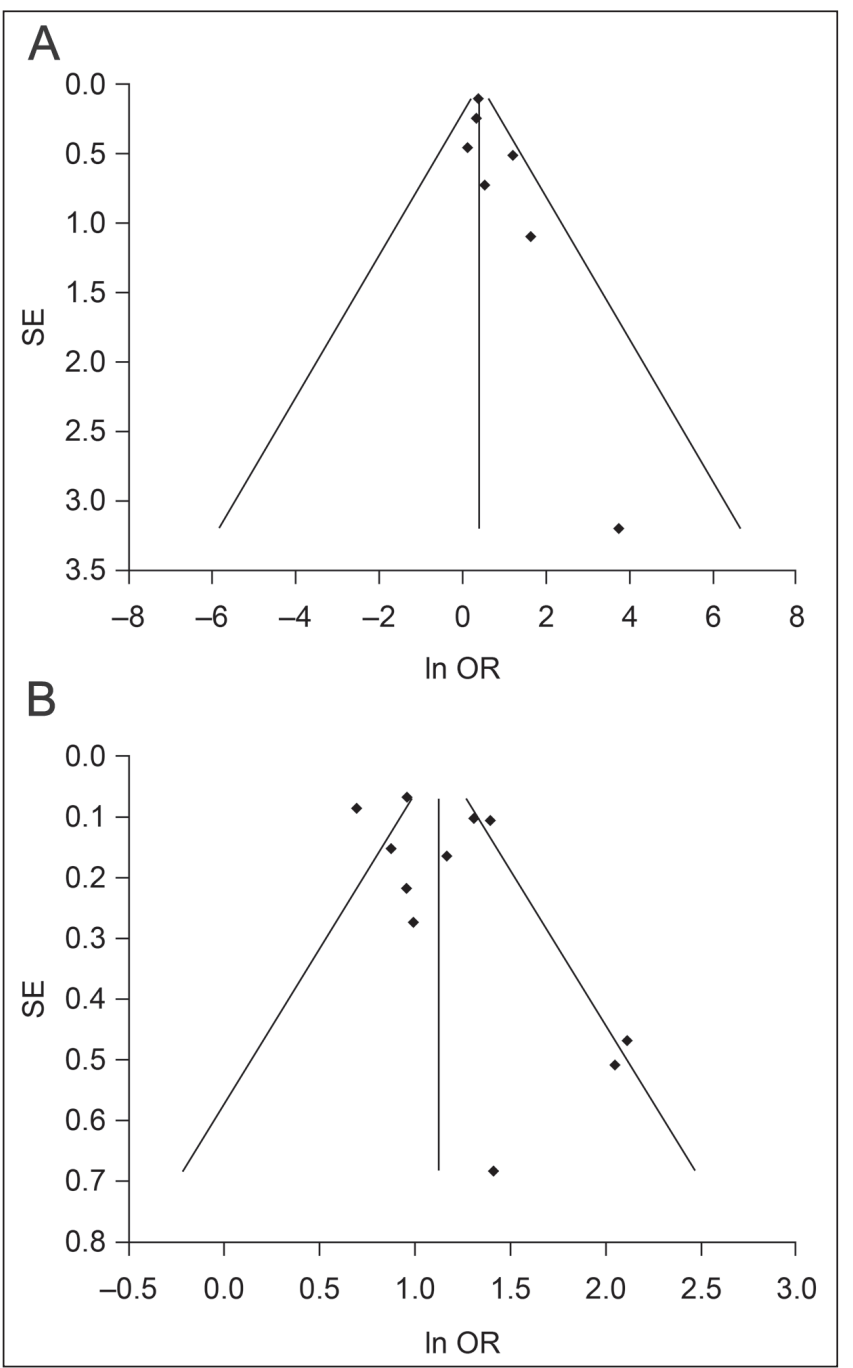

Figure 3) Funnel plot for publication bias in seven randomized controlled trials (A) and 11 observational studies (B). A Egger regression $P=0.12$ (intercept 0.8 [95\% CI -0.3 to 1.9]). B Egger regression $\mathrm{P}=0.21$ (intercept 1.6 [95\% CI -1.1 to 4.2$]$ )

remained the same as in the primary analysis (OR 1.5 [95\% CI 1.3 to 1.7]; $\mathrm{P}<0.0001$; $\mathrm{P}$ for heterogeneity $=0.57$, also using a fixed-effects model). In addition, a sensitivity analysis was performed by adding 0.5 events instead of 0.1 events to the placebo and LDA arms of the study by Silagy et al (32), which did not influence the result.

Observational studies: All 11 observational studies reported a significant increase in the rate of PUB or UGIB in individuals treated with LDA compared with those who were not treated with LDA (Figure 4).

The pooled OR of the meta-analysis of the observational studies examining the risk of UGIB associated with ASA use was 3.1 (95\% CI 2.5 to $3.7 ; \mathrm{P}<0.0001$ ) (Figure 4 ). There was a high degree of heterogeneity $\left(\mathrm{I}^{2}=78 \%\right)$; thus, a random-effects model was applied. The result of the Egger regression test for publication bias was not significant (intercept 1.6 [95\% CI -1.1 to 4.2$] ; \mathrm{P}=0.21$ ) and no funnel plot asymmetry was apparent (Figure $3 \mathrm{~B}$ ), indicating no evidence of publication bias. As mentioned in the Methods section, in the study by Uppalapati et al (31), raw outcome data were used to calculate unadjusted crude ORs, regardless of dose ( $81 \mathrm{mg} /$ day or $325 \mathrm{mg} /$ day $)$. The NNH was 819 (95\% CI 617 to 1119).

Subgroup analysis within observational studies: Two observational studies showed a trend for a greater risk of UGIB with increasing (but still low) doses of ASA $(23,46)$. Another study showed that the risk of UGIB remained the same (44). Meta-analysis of the studies examining 


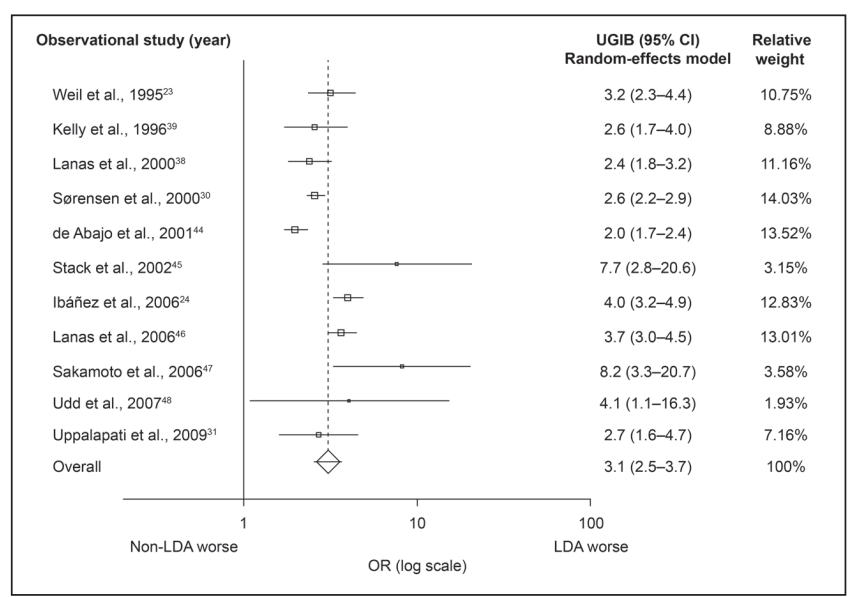

Figure 4) Meta-analysis of observational studies showing the association between low-dose acetylsalicylic acid (LDA) use and the risk of upper gastrointestinal bleeding (UGIB). For heterogeneity $P<0.001 ; I^{2}=78 \%$. Random-effects model 3.1 (95\% CI 2.5 to 3.7; P<0.0001)

the risk of UGIB associated with ASA doses $\leq 100 \mathrm{mg} /$ day found the pooled OR to be 2.6 (95\% CI 1.9 to 3.7; $\mathrm{P}<0.001$ ) (Figure 5). The OR for doses of $300 \mathrm{mg} /$ day to $325 \mathrm{mg} /$ day was 3.6 (95\% CI 1.7 to 7.3 ; $\mathrm{P}=0.001$ ) (Figure 5). A random-effects model was applied because there was a high degree of heterogeneity $\left(\mathrm{I}^{2}=76 \%\right.$ and $\mathrm{I}^{2}=90 \%$, respectively). In the cohort study, the risk was similar for users of ASA $100 \mathrm{mg} /$ day (SIR 2.6 [95\% CI 1.8 to 3.5]) and ASA $150 \mathrm{mg} /$ day (SIR 2.6 [95\% CI 2.2 to 3.0]); however, it was not clear whether ASA was used in combination with other NSAIDs.

\section{DISCUSSION}

The present meta-analysis demonstrates that use of LDA significantly increases the risk of UGIB compared with nonuse. The analysis included data from well-designed RCTs and from observational studies encompassing a broad range and large number of patients $(n=87,366)$. The pooled estimate of the RCTs yielded an OR of 1.5 (95\% CI 1.2 to $1.8 ; \mathrm{P}<0.0001)$ and the observational studies yielded an OR of $3.1(95 \%$ CI 2.5 to $3.7 ; \mathrm{P}<0.0001)$.

Although RRs are significantly increased, the absolute increases in UGIB risk are small. RCTs involving more than 22,000 patients treated with LDA show that, on average, the number of extra cases of UGIB associated with LDA use are 1.2 per 1000 patients per year. In our analysis, the NNH for the RCTs was 816 , meaning that if 816 individuals received LDA rather than placebo for one year, one would have developed UGIB as a result of treatment. The observational studies yielded a very similar NNH of 819 .

In spite of different event and/or exposure definitions, several other meta-analyses have examined the risk of gastrointestinal bleeding with LDA use $(8-16,19,20)$. In general, our findings are consistent with those of others, who reported an absolute rate increase of major gastrointestinal bleeding with LDA use of approximately one per 1000 person-years $(11,14,19)$, with a corresponding NNH of 833 (11). The RR of gastrointestinal bleeding due to ASA use has been reported to range from 1.5 to $2.5(8,10-15,19,20)$ for clinical trials, and from $2.6(9)$ to $3.3(16)$ for observational studies. A recently published meta-analysis of RCTs (20) reported a risk of major gastrointestinal bleeding (including lower gastrointestinal bleeding) with LDA use of 1.6 (95\% CI 1.3 to 1.9 ), consistent with the findings from our meta-analysis of the risk of UGIB with LDA use in RCTs. Compared with other previously published reviews and meta-analyses, our analyses included fewer studies. This was due to more stringent inclusion and data extraction criteria; to harmonize the outcome definition across studies, we only included studies in which UGIB could be clearly separated from other gastrointestinal complications such as perforation or lower gastrointestinal bleeding. To

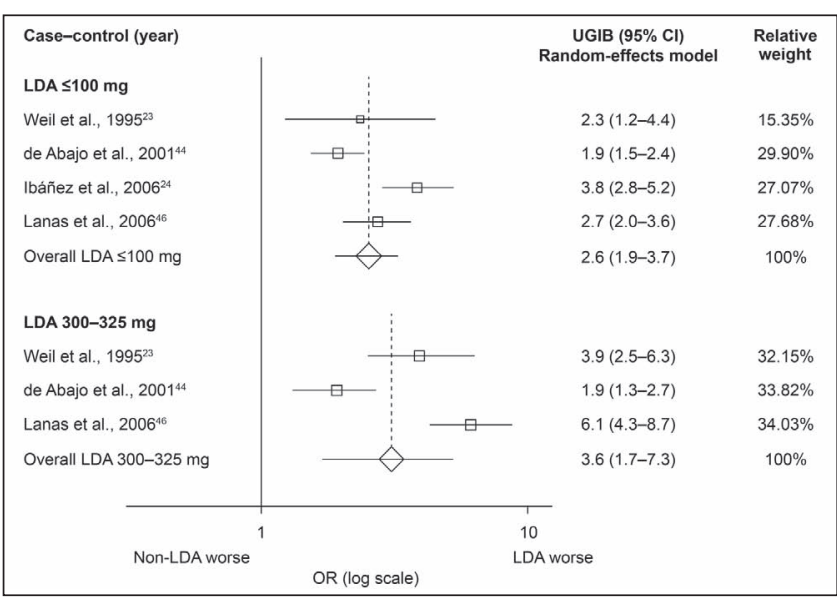

Figure 5) Meta-analysis of observational studies showing the association between low-dose acetylsalicylic acid (LDA) use and the risk of upper gastrointestinal bleeding (UGIB) stratified according to dose

harmonize the exposure definition, we included only studies in which LDA dose was clearly reported to be $<325 \mathrm{mg} /$ day. Our analysis has extended previous meta-analysis data by specifically examining low dosages of ASA only and focusing on the risk of UGIB rather than gastrointestinal bleeding in general, especially from observational data.

A potential limitation of RCTs is that they often recruit highly selected populations and, therefore, may not be representative of patients seen in routine clinical practice. For example, despite some of the RCTs included in the present analysis, which enrolled patients with a history or a high risk of cardiovascular and cerebrovascular events, all of the RCTs identified excluded certain groups of individuals who would be at an increased risk of bleeding or complications such as those with a history of peptic ulcer disease $(6,21,22,42,43)$ or individuals who were also treated with NSAIDs, anticoagulants or corticosteroids $(21,22,32)$. The risk of bleeding found in LDA users in these trials may, therefore, be lower than that in the general population. In addition, the majority of RCTs included in the present analysis were post hoc or subgroup analyses of studies in which a cardiovascular or cerebrovascular outcome (and not UGIB) was the original primary outcome measure. Thus, UGIB may have been under-reported, especially after a cardiovascular event occurred. This is supported by the finding that the risk of UGIB associated with LDA use is higher in the observational studies than in the RCTs, although it is possible that residual confounding played a role.

None of the RCTs identified in the present review tested more than one dose level of LDA. However, several of the observational studies examined the dose-response relationship between LDA and UGIB, although the results were inconsistent. For ASA doses $\leq 325 \mathrm{mg} /$ day, two studies reported an increased trend for UGIB risk with higher doses of ASA $(23,46)$. Another study showed the same risk (44) with increased ASA doses.

There is no evidence of a dose-response relationship in terms of the cardiovascular benefit of LDA, and low doses appear to be as effective as high doses (3). However, all doses of ASA are associated with an increased risk of UGIB. Therefore, there does not appear to be a 'safe' dose of ASA in terms of gastrointestinal risks. This suggests that LDA should be prescribed whenever possible, not only to ensure cardiovascular benefit, but also to reduce the risk of UGIB that is potentially associated with high doses of ASA. This is consistent with European and United States (US) guidelines, which recommend maintenance treatment with ASA $75 \mathrm{mg} /$ day to $100 \mathrm{mg} /$ day for patients with non-ST-segment elevation myocardial infarction $(49,50)$. US guidelines on reducing the gastrointestinal risks of antiplatelet therapy recommend that ASA doses $>81 \mathrm{mg} /$ day should not be used routinely (51). 
Recent studies have highlighted the importance of continuing LDA treatment, even for patients with UGIB $(52,53)$. Patients prescribed LDA for secondary prevention of cardiovascular and cerebrovascular events have a $40 \%$ increased risk of ischemic stroke or transient ischemic attack for up to six months after discontinuation of ASA treatment compared with patients who continue ASA treatment (54).

US guidelines recommend concomitant gastroprotection for patients taking LDA who are at risk for upper gastrointestinal events (eg, elderly patients, individuals using other NSAIDs or antithrombotic agents, and patients with a history of peptic ulcer disease) rather than treatment with enteric-coated or buffered LDA (51). To facilitate a more accurate estimation of the risk of UGIB, the current analysis excluded studies that reported that all participants were treated with proton pump inhibitors (PPIs). However, this guideline recommendation is supported by the finding of Ibáñez et al (24) that the risk of UGIB is lower in patients treated with LDA and a PPI than in those treated with LDA alone (24). Furthermore, there is evidence from other RCTs that PPI use decreases the incidence of peptic ulcer disease in patients treated with LDA $(55,56)$.

The present study, in contrast with previous reviews and metaanalyses, systematically evaluated the association between LDA use and UGIB, both in RCTs and in observational studies. Many previously published meta-analyses have relied on high-quality evidence from RCTs and, although observational studies have some disadvantages, such as the potential for confounding due to the nonrandomized nature of the patient groups, they allow a more representative sample of real-world data to be collected than other study designs.

Despite our efforts to harmonize the outcome definition as much as possible, potential limitations of the included studies include lack of consistency in bleeding definition and ASA dose used, which makes it difficult to compare findings across the studies. In addition, the risk estimates given in the observational studies included in the analysis are influenced by the choice of control group as, for example, in the study by Weil et al (23), in which cases were compared with hospital controls (OR 2.7 [95\% CI 1.9 to 3.80]), community controls (OR 4.2 [95\% CI 2.8 to 6.3]) or combined controls (OR 3.2 [95\% CI 2.3 to $4.4]$ ). Risk estimates in the observational studies are also dependent on the methods used to analyze the estimates of risk (eg, variations in matching and adjustment in multivariate analyses).

A potential limitation of the search strategy was the exclusion of studies published before 1989. However, some of the studies published before 1989 that were included in previous reviews would have been excluded from our study owing to other exclusion criteria, such as not separating UGIB from gastrointestinal bleeding as a whole (57) or not reporting on ASA doses $<325 \mathrm{mg} /$ day (58-60). In addition, there were many more case-control studies than cohort studies included in our review; only one cohort study was eligible for inclusion in our analysis (30). To our knowledge, the only other published systematic review of epidemiological studies of upper gastrointestinal complications and ASA use in this time period also included more case-control studies than cohort studies (9). This may have led to an underestimation of some of the results. However, it does ensure that the focus remains on recent data and that the results are likely to be as representative as possible of current clinical practice.

Another potential limitation is that it was not possible to assess precisely how many individuals in the included studies were being treated with gastroprotective medication. This may have resulted in the incidence of UGIB being underestimated.

All of the observational studies and RCTs included in these analyses show that LDA use is associated with an increased risk of UGIB. Gastroprotective prophylaxis should be considered in patients receiving long-term LDA therapy, particularly in those who have additional risk factors such as concomitant NSAID use or a history of peptic ulcer complications.

DISCLOSURE: The preparation of the manuscript was funded, in part, by AstraZeneca R\&D, Mölndal, Sweden.
ACKNOWLEDGEMENTS: Writing support was provided by Dr Rowan Pearce, from Oxford PharmaGenesis ${ }^{\mathrm{TM}}$ Ltd, Oxford, United Kingdom, and was funded by AstraZeneca R\&D, Mölndal, Sweden.

CONFLICT OF INTEREST: Vera Valkhoff, as employee of the Erasmus MC, has conducted research for AstraZeneca. Miriam Sturkenboom is head of a unit that conducts research for pharmaceutical companies (Pfizer, Lilly, AstraZeneca, and Altana). She has also acted as a consultant for Pfizer and Lundbeck. Catherine Hill is an employee of Oxford PharmaGenesis ${ }^{\mathrm{TM}}$ Ltd, which has received project funding from AstraZeneca. Sander Veldhuyzen van Zanten has received research support or speaker honoraria from, and/or served on advisory boards for, Abbott, AstraZeneca, Janssen-Ortho, Nycomed, Pfizer, and Takeda. He is a member of a data safety monitoring board of Novartis. Ernst Kuipers has served as a consultant and advisory board member for AstraZeneca.

\section{REFERENCES}

1. Anderson JL, Adams CD, Antman EM, et al. 2011 ACCF/AHA Focused Update Incorporated Into the ACC/AHA 2007 Guidelines for the management of patients with unstable angina/non-STelevation myocardial infarction: A report of the American College of Cardiology Foundation/American Heart Association Task Force on Practice Guidelines. Circulation 2011;123:2022-60.

2. Baigent C, Sudlow C, Collins R, et al. Collaborative meta-analysis of randomised trials of antiplatelet therapy for prevention of death, myocardial infarction, and stroke in high risk patients. BMJ 2002;324:71-86.

3. Björklund L, Wallander MA, Johansson S, et al. Aspirin in cardiology - benefits and risks. Int J Clin Pract 2009;63:468-77.

4. Cea Soriano L, García Rodríguez LA. Risk of upper gastrointestinal bleeding in a cohort of new users of low-dose ASA for secondary prevention of cardiovascular outcomes. Front Pharmacol 2010;1:126-35.

5. Lanas A, Scheiman J. Low-dose aspirin and upper gastrointestinal damage: Epidemiology, prevention and treatment. Curr Med Res Opin 2007;23:163-73.

6. Slattery J, Warlow CP, Shorrock CJ, et al. Risks of gastrointestinal bleeding during secondary prevention of vascular events with aspirin - analysis of gastrointestinal bleeding during the UK-TIA trial. Gut 1995;37:509-11.

7. Sprigg ER, Fleming KM, Logan RFA. Is low dose aspirin use associated with iron deficiency anaemia? An analysis of data from the Health Survey for England 2006. Gut 2009;58(Suppl II):A91.

8. Derry S, Loke YK. Risk of gastrointestinal haemorrhage with long term use of aspirin: Meta-analysis. BMJ 2000;321:1183-7.

9. García Rodríguez LA, Hernández-Díaz S, de Abajo FJ. Association between aspirin and upper gastrointestinal complications: Systematic review of epidemiologic studies. Br J Clin Pharmacol 2001;52:563-71.

10. Hayden M, Pignone M, Phillips C, et al. Aspirin for the primary prevention of cardiovascular events: A summary of the evidence for the U.S. Preventive Services Task Force. Ann Intern Med 2002;136:161-72.

11. McQuaid KR, Laine L. Systematic review and meta-analysis of adverse events of low-dose aspirin and clopidogrel in randomized controlled trials. Am J Med 2006;119:624-38.

12. Roderick PJ, Wilkes HC, Meade TW. The gastrointestinal toxicity of aspirin: An overview of randomised controlled trials. Br J Clin Pharmacol 1993;35:219-26.

13. Serebruany VL, Steinhubl SR, Berger PB, et al. Analysis of risk of bleeding complications after different doses of aspirin in 192,036 patients enrolled in 31 randomized controlled trials. Am J Cardiol 2005;95:1218-22.

14. Stalnikowicz-Darvasi R. Gastrointestinal bleeding during low-dose aspirin administration for prevention of arterial occlusive events. A critical analysis. J Clin Gastroenterol 1995;21:13-6.

15. Weisman SM, Graham DY. Evaluation of the benefits and risks of low-dose aspirin in the secondary prevention of cardiovascular and cerebrovascular events. Arch Intern Med 2002;162:2197-202.

16. Hawkey CJ. Non-steroidal anti-inflammatory drugs and peptic ulcers. BMJ 1990;300:278-84. 
17. Dickinson JP, Prentice CR. Aspirin: Benefit and risk in thromboprophylaxis. QJM 1998;91:523-38.

18. Cappelleri JC, Lau J, Kupelnick B, et al. Efficacy and safety of different aspirin dosages on vascular diseases in high-risk patients. A metaregression analysis. Online J Curr Clin Trials 1995; Doc No 174.

19. Sudlow C. Cardiovascular disorders: Primary prevention. Clinical evidence. American College of Physicians-American Society of Internal Medicine, 5th edn. London: BMJ Publishing Group, 2001.

20. Lanas A, Wu P, Medin J, et al. Low doses of acetylsalicylic acid increase risk of gastrointestinal bleeding in a meta-analysis. Clin Gastroenterol Hepatol 2011;9:762-8.

21. Final report on the aspirin component of the ongoing Physicians' Health Study. Steering Committee of the Physicians' Health Study Research Group. N Engl J Med 1989;321:129-35.

22. Diener HC, Forbes C, Riekkinen PJ, et al. European stroke prevention study 2: Efficacy and safety data. J Neurol Sci 1997;151:S41-S51.

23. Weil J, Colin-Jones D, Langman M, et al. Prophylactic aspirin and risk of peptic ulcer bleeding. BMJ 1995;310:827-30.

24. Ibáñez L, Vidal X, Vendrell L, et al. Upper gastrointestinal bleeding associated with antiplatelet drugs. Aliment Pharmacol Ther 2006;23:235-42.

25. Diener HC, Cunha L, Forbes C, et al. European Stroke Prevention Study. 2. Dipyridamole and acetylsalicylic acid in the secondary prevention of stroke. J Neurol Sci 1996;143:1-13.

26. Langman MJ, Weil J, Wainwright P, et al. Risks of bleeding peptic ulcer associated with individual non-steroidal anti-inflammatory drugs. Lancet 1994;343:1075-8.

27. Laporte JR, Ibáñez L, Vidal X, et al. Upper gastrointestinal bleeding associated with the use of NSAIDs: Newer versus older agents. Drug Saf 2004;27:411-20.

28. Farrell B, Godwin J, Richards S, et al. The United Kingdom transient ischaemic attack (UK-TIA) aspirin trial: Final results. J Neurol Neurosurg Psychiatry 1991;54:1044-54.

29. Bjerre LM, LeLorier J. Expressing the magnitude of adverse effects in case-control studies: The number of patients needed to be treated for one additional patient to be harmed. BMJ 2000;320:503-6.

30. Sørensen HT, Mellemkjær L, Blot WJ, et al. Risk of upper gastrointestinal bleeding associated with use of low-dose aspirin. Am J Gastroenterol 2000;95:2218-24.

31. Uppalapati SS, Boylan JD, Stoltzfus J. Risk factors involved in patients with bleeding peptic ulcers: A case-control study. Dig Dis Sci 2009;54:593-8.

32. Silagy CA, McNeil JJ, Donnan GA, et al. Adverse effects of low-dose aspirin in a healthy elderly population. Clin Pharmacol Ther 1993;54:84-9.

33. Bax L, Yu LM, Ikeda N, et al. Development and validation of MIX: Comprehensive free software for meta-analysis of causal research data. BMC Med Res Methodol 2006;6:50.

34. Egger M, Davey Smith G, Schneider M, et al. Bias in meta-analysis detected by a simple, graphical test. BMJ 1997;315:629-34.

35. Lanas A, Bajador E, Serrano P, et al. Effects of nitrate and prophylactic aspirin on upper gastrointestinal bleeding: A retrospective case-control study. J Int Med Res 1998;26:120-8.

36. Santolaria S, Lanas A, Benito R, et al. Helicobacter pylori infection is a protective factor for bleeding gastric ulcers but not for bleeding duodenal ulcers in NSAID users. Aliment Pharmacol Ther 1999;13:1511-8.

37. Kaufman DW, Kelly JP, Sheehan JE, et al. Nonsteroidal antiinflammatory drug use in relation to major upper gastrointestinal bleeding. Clin Pharmacol Ther 1993;53:485-94.

38. Lanas A, Bajador E, Serrano P, et al. Nitrovasodilators, low-dose aspirin, other nonsteroidal anti-inflammatory drugs, and the risk of upper gastrointestinal bleeding. N Engl J Med 2000;343:834-9.

39. Kelly JP, Kaufman DW, Jurgelon JM, et al. Risk of aspirin-associated major upper-gastrointestinal bleeding with enteric-coated or buffered product. Lancet 1996;348:1413-6.

40. Kaufman DW, Kelly JP, Wiholm BE, et al. The risk of acute major upper gastrointestinal bleeding among users of aspirin and ibuprofen at various levels of alcohol consumption. Am J Gastroenterol 1999;94:3189-96.
41. The Medical Research Council's General Practice Research Framework. Thrombosis prevention trial: Randomised trial of low-intensity oral anticoagulation with warfarin and low-dose aspirin in the primary prevention of ischaemic heart disease in men at increased risk. Lancet 1998;351:233-41.

42. Prevention of pulmonary embolism and deep vein thrombosis with low dose aspirin: Pulmonary Embolism Prevention (PEP) trial. Lancet 2000;355:1295-302.

43. Ogawa H, Nakayama M, Morimoto T, et al. Low-dose aspirin for primary prevention of atherosclerotic events in patients with type 2 diabetes: A randomized controlled trial. JAMA 2008;300:2134-41.

44. de Abajo FJ, García Rodríguez LA. Risk of upper gastrointestinal bleeding and perforation associated with low-dose aspirin as plain and enteric-coated formulations. BMC Clin Pharmacol 2001;1:1.

45. Stack WA, Atherton JC, Hawkey GM, et al. Interactions between Helicobacter pylori and other risk factors for peptic ulcer bleeding. Aliment Pharmacol Ther 2002;16:497-506.

46. Lanas A, García Rodríguez LA, Arroyo MT, et al. Risk of upper gastrointestinal ulcer bleeding associated with selective cyclooxygenase-2 inhibitors, traditional non-aspirin non-steroidal antiinflammatory drugs, aspirin and combinations. Gut 2006;55:1731-8.

47. Sakamoto C, Sugano K, Ota S, et al. Case-control study on the association of upper gastrointestinal bleeding and nonsteroidal antiinflammatory drugs in Japan. Eur J Clin Pharmacol 2006;62:765-72.

48. Udd M, Miettinen P, Palmu A, et al. Analysis of the risk factors and their combinations in acute gastroduodenal ulcer bleeding: A case-control study. Scand J Gastroenterol 2007;42:1395-403.

49. Becker RC, Meade TW, Berger PB, et al. The primary and secondary prevention of coronary artery disease: American College of Chest Physicians Evidence-Based Clinical Practice Guidelines, 8th edn. Chest 2008;133:776S-814S.

50. Hamm CW, Bassand JP, Agewall S, et al. ESC Guidelines for the management of acute coronary syndromes in patients presenting without persistent ST-segment elevation: The Task Force for the management of acute coronary syndromes (ACS) in patients presenting without persistent ST-segment elevation of the European Society of Cardiology (ESC). Eur Heart J 2011;32:2999-3054.

51. Bhatt DL, Scheiman J, Abraham NS, et al. ACCF/ACG/AHA 2008 expert consensus document on reducing the gastrointestinal risks of antiplatelet therapy and NSAID use: A report of the American College of Cardiology Foundation Task Force on Clinical Expert Consensus Documents. Circulation 2008;118:1894-909.

52. Sung JJ, Lau JY, Ching JY et al. Continuation of low-dose aspirin therapy in peptic ulcer bleeding: A randomized trial. Ann Intern Med 2010;152:1-9.

53. García Rodríguez LA, Cea Soriano L, Johansson S. Increased risk of stroke after discontinuation of low-dose acetylsalicylic acid therapy: A UK primary care study. Cerebrovasc Dis 2010;29 Suppl 2:79.

54. García Rodríguez LA, Cea Soriano L, Hill C, et al. Increased risk of stroke after discontinuation of acetylsalicylic acid: A UK primary care study. Neurology 2011;76:740-6.

55. Yeomans N, Lanas A, Labenz J, et al. Efficacy of esomeprazole (20 mg once daily) for reducing the risk of gastroduodenal ulcers associated with continuous use of low-dose aspirin.

Am J Gastroenterol 2008;103:2465-73.

56. Scheiman J, Herlitz J, Agewall S, et al. Peptic ulcer prevention by esomeprazole $20 \mathrm{mg}$ and $40 \mathrm{mg}$ once daily in patients taking lowdose acetylsalicylic acid for secondary cardiovascular prevention. Gut 2010;59:A53.

57. Lewis HD Jr. Unstable angina: Status of aspirin and other forms of therapy. Circulation 1985;72:V155-60.

58. Peto R, Gray R, Collins R, et al. Randomised trial of prophylactic daily aspirin in British male doctors. BMJ (Clin Res Ed) 1988;296:313-16.

59. Levy M. Aspirin use in patients with major upper gastrointestinal bleeding and peptic-ulcer disease. A report from the Boston Collaborative Drug Surveillance Program, Boston University Medical Center. N Engl J Med 1974;290:1158-62.

60. Levy M, Miller DR, Kaufman DW, et al. Major upper gastrointestinal tract bleeding. Relation to the use of aspirin and other nonnarcotic analgesics. Arch Intern Med 1988;148:281-5. 


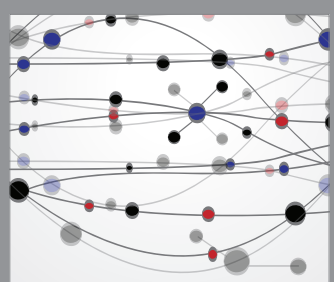

The Scientific World Journal
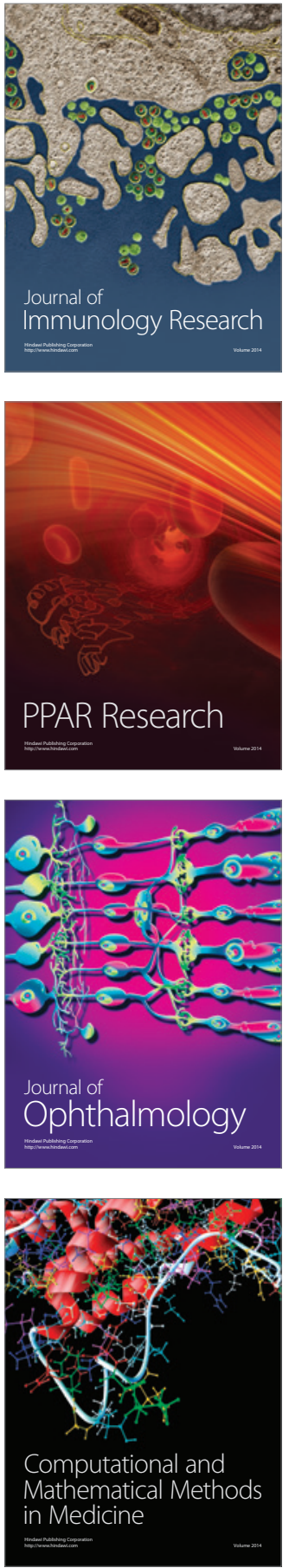

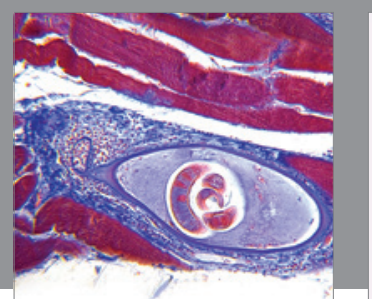

Gastroenterology Research and Practice

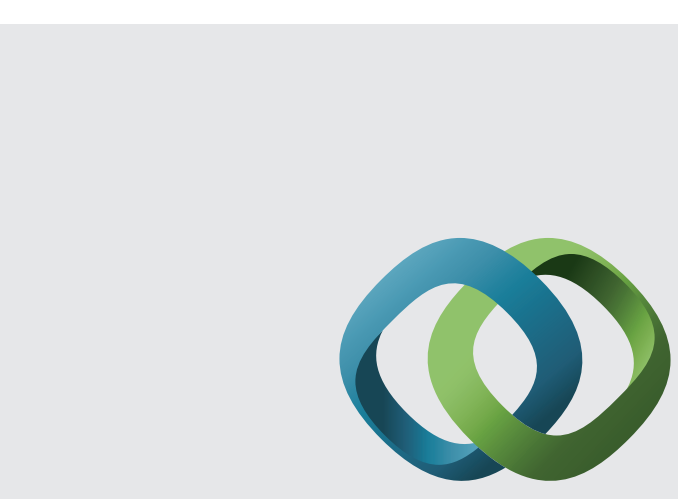

\section{Hindawi}

Submit your manuscripts at

http://www.hindawi.com
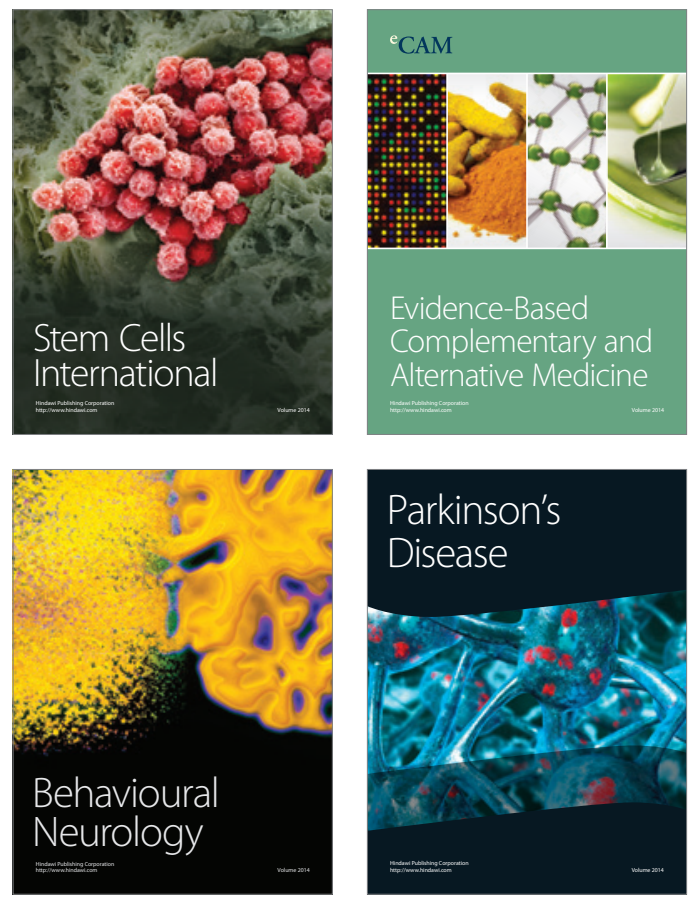
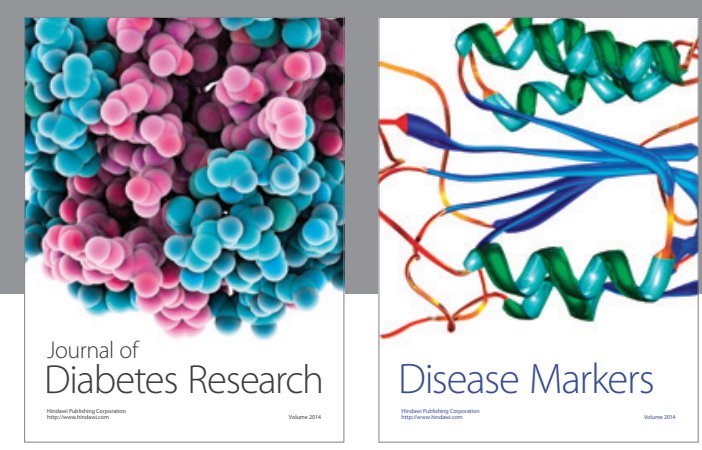

Disease Markers
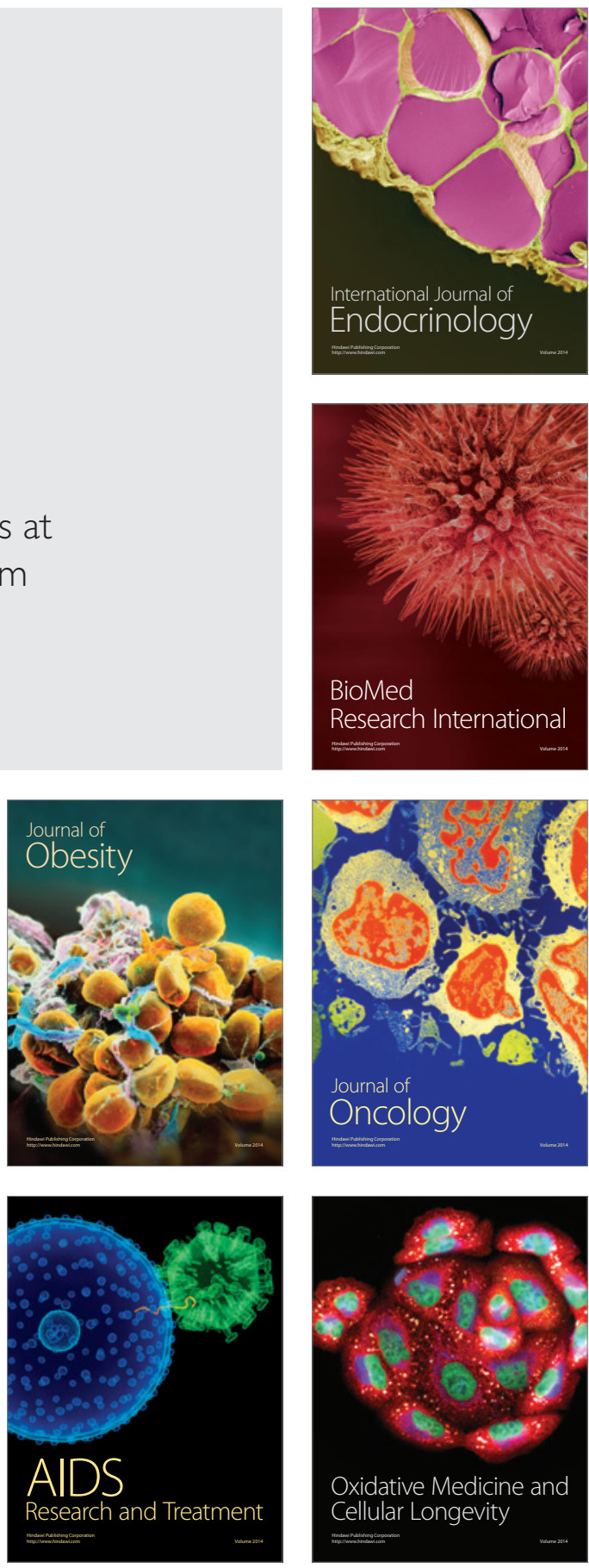